\title{
Deletion Rules for Equivalent Sequential and Parallel Reductions
}

\author{
Kálmán Palágyi \\ Department of Image Processing and Computer Graphics, \\ University of Szeged, Hungary \\ palagyi@inf.u-szeged.hu
}

\begin{abstract}
A reduction operator transforms a binary picture only by changing some black points to white ones, which is referred to as deletion. Sequential reductions may delete just one point at a time, while parallel reductions can alter a set of points simultaneously. Two reductions are called equivalent if they produce the same result for each input picture. This work lays a bridge between the parallel and the sequential strategies. A class of deletion rules are proposed that provide 2D parallel reductions being equivalent to sequential reductions. Some new sufficient conditions for topology-preserving parallel reductions are also reported.
\end{abstract}

Keywords: Discrete Geometry, Digital Topology, Topology-Preserving Reductions.

\section{Introduction}

A binary picture on the 2-dimensional digital space $\mathbb{Z}^{2}$ is a mapping that assigns a color of black or white to each point of $\mathbb{Z}^{2}[6]$. A reduction (or reductive [3]) operator transforms a binary picture only by changing some black points to white ones, which is referred to as the deletion of 1s. Reductions play important role in various topological algorithms, e.g., thinning [2/11] or shrinking [3].

Parallel reductions can alter a set of points simultaneously, while sequential reductions may delete just one black point at a time. Parallel reductions and sequential reductions strategies are illustrated by Algorithm 1 and Algorithm 2, respectively.

By comparing the two approaches, we can state that in the parallel case (see Algorithm 1) the initial set of black points is considered when the deletion rule is evaluated for each elements of the set of interesting points $X$. On the contrary, the set of black points is dynamically altered during the sequential reduction (see Algorithm 2).

Algorithms 1 and 2 consider a set of interesting points $X$ and its complementary $C$ that is called a constraint set. Constraint sets may contain some types of border points in subiteration-based (or directional) algorithms or points that are not in the activated subfields in the case of subfield-based algorithms [2. In addition, endpoints (i.e., some points that provide important geometrical information 

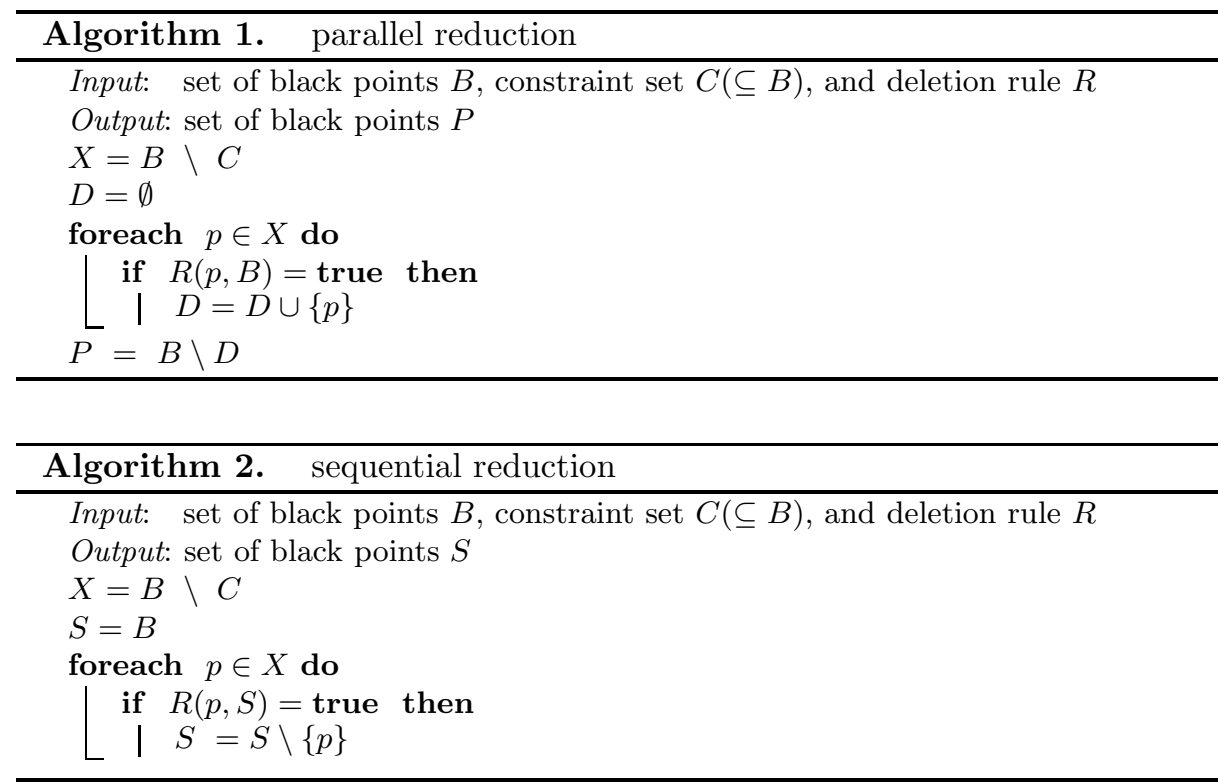

relative to the shape of the objects [2]) or isthmuses (i.e., some curve/surface interior points [1] can also be accumulated in $C$.

Two reductions are said to be equivalent if they produce the same result for each input binary picture. A deletion rule is called equivalent if it yields a pair of equivalent parallel and sequential reductions.

The rule $R(p, Y)$ says that a point $p$ in question is deletable or not in a set of black points $Y$. Note that elements in $C$ are omitted when the deletion rule $R$ is evaluated.

The sequential approach suffers from the drawback that different visiting order of interesting points may yield various results. Order-independent sequential reductions can produce the same result for any visiting order of the elements in $X$ [49]. It is clear that only order-independent sequential reductions can be equivalent to parallel ones.

In this paper we establish some conditions for deletion rules that provide equivalent parallel and sequential reductions. Some new sufficient conditions for topology-preserving parallel reductions are also proposed.

\section{Basic Notions and Results}

In this paper, we use the fundamental concepts of digital topology as reviewed by Kong and Rosenfeld [6].

Let $p$ be a point in the 2-dimensional digital space $\mathbb{Z}^{2}$. Let us denote $N_{m}(p)$ the set of points that are $m$-adjacent to $p$ and let $N_{m}^{*}(p)=N_{m}(p) \backslash\{p\}(m=4,8)$, 


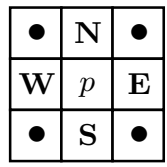

Fig. 1. The considered adjacency relations in $\mathbb{Z}^{2}$. The set $N_{4}(p)$ contains point $p$ and the four points marked "N", "E", "S", and "W". The set $N_{8}(p)$ contains $N_{4}(p)$ and the additional four points marked "•".

see Fig. 1] Note that, throughout this paper, all figures are depicted on the square grid that is dual to $\mathbb{Z}^{2}$.

The equivalence classes relative to the $m$-connectivity relation (i.e., the reflexive and transitive closure of the $m$-adjacency) are the $m$-components of a set of points $X \subseteq \mathbb{Z}^{2}$.

A $(8,4)$ digital picture $\mathcal{P}$ is a quadruple $\left(\mathbb{Z}^{2}, 8,4, B\right)$. Each element of $\mathbb{Z}^{2}$ is said to be a point of $\mathcal{P}$. Each point in $B \subseteq \mathbb{Z}^{2}$ is called a black point. Each point in $\mathbb{Z}^{2} \backslash B$ is said to be a white point. An object is an 8-component of $B$, while a white component is a 4-component of $\mathbb{Z}^{2} \backslash B$.

A picture $\left(\mathbb{Z}^{2}, 8,4, B\right)$ is called finite if the set $B$ contains finitely many points. In a finite picture there is a unique infinite white component, which is called the background. A finite white component is said to be a cavity.

A black point is called a border point in a $(8,4)$ picture if it is 4 -adjacent to at least one white point. A border point $p$ is said to be an $\mathbf{N}$-border point if the point marked "N" in Fig. 1 is white. We can define $\mathbf{E}-, \mathbf{S}_{-}$, and $\mathbf{W}$-border points in the same way. A black point in a picture is called an interior point if it is not a border point.

A 2D reduction is not topology-preserving if any object in the input picture is split (into several ones) or is completely deleted, any cavity in the input picture is merged with the background or another cavity, or a cavity is created where there was none in the input picture [5].

A black point is simple in a picture if and only if its deletion is a topologypreserving reduction [6]. We state now the following characterization of simple points of $(8,4)$ pictures:

Theorem 1. 6] Black point $p$ is simple in a picture $\left(\mathbb{Z}^{2}, 8,4, B\right)$ if and only if all of the following conditions hold:

1. The set $N_{4}^{*}(p) \cap B$ contains exactly one 8-component.

2. $N_{4}(p) \backslash B \neq \emptyset$.

Condition 2 of Theorem 1 means that only borders points may be simple points. Hence interior points are not simple points.

Reductions generally delete a set of black points and not just a single simple point. Hence we need to consider what is meant by topology preservation when a number of black points are deleted simultaneously. Various authors proposed some sufficient conditions for reductions to preserve topology [5 810]. 


\section{Conditions for Equivalent Deletion Rules}

Recall that a deletion rule is equivalent if it determines equivalent parallel and (order-independent) sequential reductions (see Algorithms 1 and 2). In this section some sufficient conditions for equivalent deletion rules are introduced.

Definition 1. Let $R$ be the deletion rule. Let $\left(\mathbb{Z}^{2}, 8,4, B\right)$ be an arbitrary picture, and let $q \in B$ be any point that is deleted from that picture by $R$. Deletion rule $R$ is general if the following conditions hold:

1. If $p$ can be deleted from picture $\left(\mathbb{Z}^{2}, 8,4, B\right)$ by $R$, then $p$ can be deleted from picture $\left(\mathbb{Z}^{2}, 8,4, B \backslash\{q\}\right)$ by $R$.

2. If $p$ cannot be deleted from picture $\left(\mathbb{Z}^{2}, 8,4, B\right)$ by $R$, then $p$ cannot be deleted from picture $\left(\mathbb{Z}^{2}, 8,4, B \backslash\{q\}\right)$ by $R$.

Let us state some useful properties of general deletion rules.

Lemma 1. Each sequential reduction with a general deletion rule is order-independent.

Proof. Assume that a sequential reduction with a general deletion rule $R$ produces different results for distinct visiting orders of the set $X$ with $k$ elements. It is obvious that $k \geq 2$ holds. All permutations of the elements of $X$ can be get by swapping a pair of successive points. (Recall the bubble sort algorithm.) Let us consider the following two permutations

$$
\begin{aligned}
& \Pi_{1}(X)=\left\langle x_{1}, \ldots, x_{i-1}, p, q, x_{i+2}, \ldots, x_{k}\right\rangle \text { and } \\
& \Pi_{2}(X)=\left\langle x_{1}, \ldots, x_{i-1}, q, p, x_{i+2}, \ldots, x_{k}\right\rangle,
\end{aligned}
$$

such that different results are produced.

Let $\left(\mathbb{Z}^{2}, 8,4, S\right)$ be the interim picture when point $p$ is visited according to $\Pi_{1}(X)$ (i.e., when $q$ is investigated by $\Pi_{2}(X)$ ). (Note that both $p$ and $q$ are black points in that picture.)

Then we need to take the following two points into consideration:

1. Point $p$ can be deleted from picture $\left(\mathbb{Z}^{2}, 8,4, S\right)$ by $R$, but $p$ cannot be deleted from picture $\left(\mathbb{Z}^{2}, 8,4, S \backslash\{q\}\right)$ by $R$.

2. Point $p$ cannot be deleted from picture $\left(\mathbb{Z}^{2}, 8,4, S\right)$ by $R$, but $p$ can be deleted from picture $\left(\mathbb{Z}^{2}, 8,4, S \backslash\{q\}\right)$ by $R$.

Note that $q$ can be deleted from picture $\left(\mathbb{Z}^{2}, 8,4, S\right)$ by $R$.

Since $R$ is general, in both cases we arrived at contradictions. Hence deletion rule $R$ produces the same result for any visiting orders.

Lemma 2. Let $R$ be a general deletion rule. Then the parallel and the sequential reductions with $R$ are equivalent. 
Proof. We need to show that both cases produce the same result for any input picture. In other words, $P=S$ when Algorithms 1 and 2 terminate.

Let $p \in X \cap P$ (i.e., $R(p, B)=$ false). Since the sequential reduction is orderindependent by Lemma 1 , we can assume that point $p$ is visited first. Then deletability of $p$ is evaluated in the initial set of black points $S=B$. In this case $p \in X \cap S$ since $R(p, S)=R(p, B)=$ false. $p \in S \cap(B \backslash X)$ stands for each $p \in P \cap(B \backslash X)$, thus $P \subseteq S$.

Conversely, $S \subseteq P$ can be seen in the same way. Hence $P=S$.

We are ready to state a condition for equivalent deletion rules as an easy consequence of Lemma 2.

Theorem 2. A deletion rule is equivalent if it is general.

The following theorem provides some new sufficient conditions for topologypreserving reductions.

Theorem 3. A (parallel) reduction $\mathcal{R}$ is topology-preserving if the following conditions hold:

1. The deletion rule $R$ associated with $\mathcal{R}$ is general.

2. $R$ deletes only simple points.

Proof. If the deletion rule of a sequential reduction may delete a simple point, then the entire sequential reduction with the same rule is topology-preserving. Since $R$ is general, the sequential reduction is equivalent to $\mathcal{R}$ by Theorem 2 . Hence $\mathcal{R}$ is topology-preserving.

Figure 2 presents an example of a non-general deletion rule.

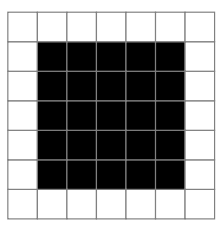

(a)

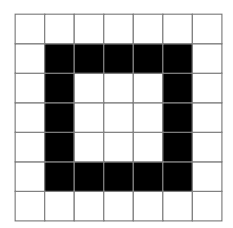

(b)

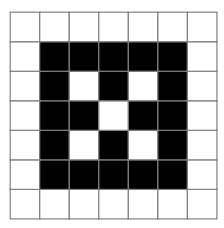

(c)

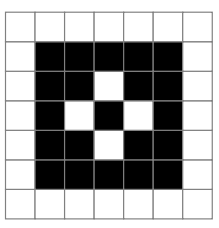

(d)

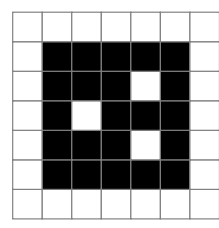

(e)

Fig. 2. Example of a non-general deletion rule that removes interior points. We can state that the parallel and the sequential reductions with that rule cannot produce the same result for the original object (a). The result produced by the parallel reduction (b). Three of the possible results produced by the sequential reduction with various visiting orders (c)-(e). 


\section{Example of an Equivalent Deletion Rule}

In this section a general and topology-preserving reduction is presented. Hence it is equivalent to a (topology-preserving and order-independent) sequential reduction.

Consider the deletion rule $R$ that is given by the set of 9 matching templates $\mathcal{T}$ depicted in Fig. 3. A black point is deleted by $R$ if at least one template in $\mathcal{T}$ matches it. The constraint set $C$ (see Algorithms 1 and 2) assigned to $R$ is defined as follows:

$$
C=\{p \mid p \text { is not an } \mathbf{N} \text {-border point in } B\} .
$$
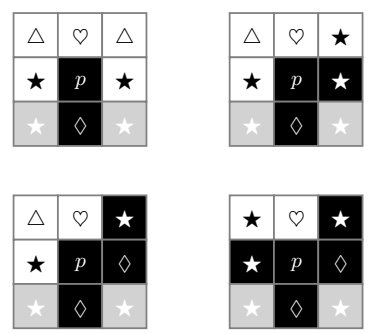
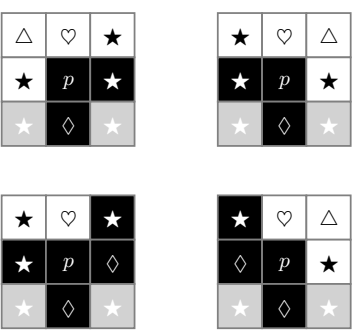
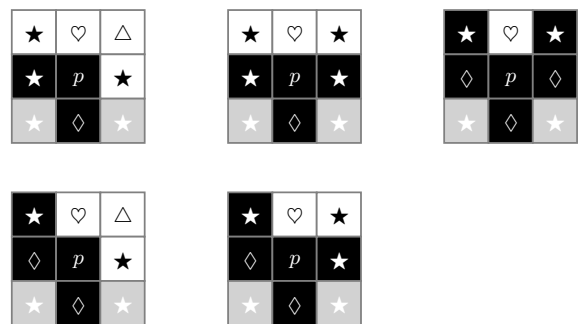

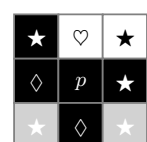

Fig. 3. The set of 9 matching templates $\mathcal{T}$ associated with the deletion rule $R$. The central point $p$ is matched by a template if each black position matches a black point and each white element matches a white point. The template position depicted in grey matches either a black or a white point.

Let us state some properties of $R$.

Proposition 1. Each deletable point is an $\mathbf{N}$-border point and not an $\mathbf{S}$-border point.

Proposition 2. If we alter any position marked “ $\star$ " in a template in $\mathcal{T}$, we get a template in $\mathcal{T}$.

Proposition 3. If we alter a position marked " $\triangleright$ " or " $\triangle$ " in a template in $\mathcal{T}$, we do not get a template in $\mathcal{T}$.

Propositions 1-3 are obvious by careful examination of the templates in $\mathcal{T}$.

Proposition 4. Black points marked " $\diamond$ " cannot be deleted by $R$ (see Fig. 3).

It is obvious by Proposition 1 since those points are not $\mathbf{N}$-border points. Note that all those points are in the constraint set $C$.

Proposition 5. All non-central black and grey positions are marked " $\diamond$ " or " " in each template in $\mathcal{T}$. 
Proposition 6. Simple points are deleted by $R$.

It is easy to see that both conditions of Theorem 1 hold for each point that is matched by a template in $\mathcal{T}$.

We are ready to state the following theorem.

Theorem 4. Deletion rule $R$ with respect to the constraint set $C$ is general.

Proof. Let $\left(\mathbb{Z}^{2}, 8,4, B\right)$ be an arbitrary picture. To prove this theorem we must show that the following two conditions are satisfied for any point $q \in B$ that is deleted by $R$ :

1. If $p$ can be deleted from picture $\left(\mathbb{Z}^{2}, 8,4, B\right)$ by $R$, then $p$ can be deleted from picture $\left(\mathbb{Z}^{2}, 8,4, B \backslash\{q\}\right)$ by $R$.

2. If $p$ cannot be deleted from picture $\left(\mathbb{Z}^{2}, 8,4, B\right)$ by $R$, then $p$ cannot be deleted from picture $\left(\mathbb{Z}^{2}, 8,4, B \backslash\{q\}\right)$ by $R$.

Since $R$ is defined by $3 \times 3$ templates, there is nothing to prove if $q \notin N_{8}^{*}(p)$. Assume that $p$ can be deleted from picture $\left(\mathbb{Z}^{2}, 8,4, B\right)$ by $R$. Then at least one template in $\mathcal{T}$ matches it and point $q \in N_{8}^{*}(p)$ is an element depicted in black or grey in that template. Since $q$ can be deleted, it cannot be marked " $\vee$ " by Proposition 4. Then $q$ is marked " $\star$ " by Proposition 5 . Hence $p$ can be deleted from picture $\left(\mathbb{Z}^{2}, 8,4, B \backslash\{q\}\right)$ by Proposition 2 .

To verify the second case, assume that $p$ cannot be deleted from picture $\left(\mathbb{Z}^{2}, 8,4, B\right)$. Then $p$ is matched by a template $T^{\prime} \notin \mathcal{T}$. It is easy to see that $T^{\prime}$ can be derived from a template $T \in \mathcal{T}$ by altering a white position marked " $\triangleright$ " or " $\triangle$ " by Proposition 3.

- If a white position marked " $\odot$ " is altered in template $T$, then point $p$ is not an $\mathbf{N}$-border point in picture $\left(\mathbb{Z}^{2}, 8,4, B\right)$. Hence $p \in C$ and it cannot be deleted by $R$.

- If a white position marked " $\triangle$ " is altered in template $T$, then point $q$ cannot be deleted by Proposition 1 . Since $q$ is deleted by $R$, we arrived at a contradiction.

Theorems 2 and 4 together imply that the deletion rule $R$ is equivalent. In addition the (parallel) reduction associated with $R$ is topology-preserving by Proposition 6 and Theorem 3.

Note that $R$ may be the base rule of a 4 -subiteration 2D parallel thinning algorithm. The deletion rules of the remaining three subiterations are the rotated versions of the rule associated with the deletion direction $\mathbf{N}$.

\section{Conclusions}

This work lays a bridge between the parallel and the sequential reductions. A class of deletion rules are proposed to determine equivalent $2 \mathrm{D}$ parallel and 
order-independent sequential reductions. A new sufficient condition for topologypreserving reductions is also reported. It provides a method of verifying that a parallel algorithm always preserves topology.

We are going to extend this work to the case of 3D $(26,6)$ pictures, and find $3 \mathrm{D}$ deletion rules for equivalent parallel and sequential reductions. We also plan to construct subiteration-based and subfield-based parallel thinning algorithms that produce the same result for any order of deletion directions and subfields.

Acknowledgements. This work was supported by the European Union and co-funded by the European Social Fund. Project title: "Telemedicine-focused research activities on the field of Mathematics, Informatics and Medical sciences." Project number: TÁMOP-4.2.2.A-11/1/KONV-2012-0073.

The author would like to thank the anonymous reviewers for their valuable suggestions and remarks.

\section{References}

1. Bertrand, G., Couprie, M.: Transformations topologiques discrètes. In: Coeurjolly, D., Montanvert, A., Chassery, J. (eds.) Géométrie Discrète et Images Numériques, pp. 187-209. Hermès Science Publications (2007)

2. Hall, R.W.: Parallel connectivity-preserving thinning algorithms. In: Kong, T.Y., Rosenfeld, A. (eds.) Topological Algorithms for Digital Image Processing, pp. 145179. Elsevier Science B.V (1996)

3. Hall, R.W., Kong, T.Y., Rosenfeld, A.: Shrinking binary images. In: Kong, T.Y., Rosenfeld, A. (eds.) Topological Algorithms for Digital Image Processing, pp. 3198. Elsevier Science B.V (1996)

4. Kardos, P., Palágyi, K.: Order-independent sequential thinning in arbitrary dimensions. In: Proc. Int. Conf. Signal and Image Processing and Applications, SIPA 2011, pp. 129-134 (2011)

5. Kong, T.Y.: On topology preservation in 2-d and 3-d thinning. Int. Journal of Pattern Recognition and Artificial Intelligence 9, 813-844 (1995)

6. Kong, T.Y., Rosenfeld, A.: Digital topology: Introduction and survey. Computer Vision, Graphics, and Image Processing 48, 357-393 (1989)

7. Lam, L., Lee, S.-W., Suen, S.-W.: Thinning methodologies - A comprehensive survey. IEEE Trans. Pattern Analysis and Machine Intelligence 14, 869-885 (1992)

8. Németh, G., Palágyi, K.: Topology preserving parallel thinning algorithms. International Journal of Imaging Systems and Technology 23, 37-44 (2011)

9. Ranwez, V., Soille, P.: Order independent homotopic thinning for binary and grey tone anchored skeletons. Pattern Recognition Letters 23, 687-702 (2002)

10. Ronse, C.: Minimal test patterns for connectivity preservation in parallel thinning algorithms for binary digital images. Discrete Applied Mathematics 21, 67-79 (1988)

11. Suen, C.Y., Wang, P.S.P. (eds.): Thinning methodologies for pattern recognition. Series in Machine Perception and Artificial Intelligence, vol. 8. World Scientific (1994) 